\title{
On the Uniform Continuity of the Probabilistic Distance
}

\author{
B. SCHWEIZER
}

\section{Received October 8, 1965}

It is a basic fact that in a metric space $(S, d)$ the distance function $d$ is a uniformly continuous mapping from $S \times S$ into the non-negative real numbers. This result is an immediate consequence of the triangle inequality. The topology on $S$ is the metric topology, the topology on $S \times S$ is the induced product topology and the topology on the non-negative reals is the usual one. In probabilistic metric spaces $[3,5,6]$, where the distances between points are probability distribution functions rather than numbers and where a variety of triangle inequalities is possible, the situation is more involved. The connections between triangle inequalities and continuity properties of the probabilistic distance were first studied in [5]. The results obtained there, however, are not optimal. In this paper we return to the question and prove a theorem which is without doubt the natural extension to probabilistic metric spaces of the metric space theorem mentioned above. To this end we first recall several definitions and results from the theory of probabilistic metric spaces.

A probabilistic metric space (briefly, a $P M$ space) is an ordered pair $(S, \mathscr{J})$, where $S$ is a set and $\mathscr{J}$ a mapping from $S \times S$ into the set of one-dimensional distribution functions. For any pair of points $p, q$ of $S$, the distribution function $\mathscr{J}(p, q)$ is denoted by $F_{p q}$; for any real number $x$, the number $F_{p q}(x)$ is usually interpreted as the probability that the "distance" between $p$ and $q$ is less than $x$. The functions $F_{p q}$ are assumed to satisfy the following conditions:

I. $F_{p q}(x)=1$ for all $x>0$ if and only if $p=q$;

II. $F_{p q}(0)=0$;

III. $\quad F_{p q}=F_{q p}$;

IV. If $F_{p q}(x)=1$ and $F_{q r}(y)=1$, then $F_{p r}(x+y)=1$.

A $t$-norm $T$ is a two-place function from the closed unit square to the closed unit interval satisfying the conditions:

(a) $T(0,0)=0, T(a, 1)=a$;

(b) $T(c, d) \geqq T(a, b)$ whenever $c \geqq a, d \geqq b$;

(c) $T(a, b)=T(b, a)$;

(d) $T(a, T(b, c))=T(T(a, b), c)$.

A $t$-norm $T$ is stronger than a $t$-norm $T^{\prime}$ if $T(a, b) \geqq T^{\prime}(a, b)$ for all $(a, b)$ in the unit square and strict inequality holds for at least one pair $(a, b)$.

A Menger space is an ordered triple $(S, \mathscr{J}, T)$ such that $(S, \mathscr{J})$ is a PM space, $T$ is a $t$-norm, and the generalized triangle inequality

IVm.

$$
F_{p r}(x+y) \geqq T\left(F_{p q}(x), F_{q r}(y)\right)
$$

holds for all triples of points $p, q, r$ in $S$ and for all $x, y \geqq 0$. 
Finally, if $(S, \mathscr{J}, T)$ is a Menger space under a $t$-norm $T$ such that

$$
\lim _{x \rightarrow 1} T(a, x)=a
$$

for every $a$ between 0 and 1 , then the system of neighborhoods defined by

$$
N_{p}(\varepsilon, \lambda)=\left\{q \mid F_{p q}(\varepsilon)>1-\lambda, \varepsilon>0, \lambda>0\right\}
$$

is a neighborhood system for a (metrizable) topology on $S$, called the $\varepsilon, \lambda$-topology $[4,5]$.

In addition to the above facts concerning PM spaces, we also need the fact that the function $L$ defined for any pair of distribution functions $F$ and $G$ by

$$
L(F, G)=\inf \{h \mid F(x-h)-h \leqq G(x) \leqq F(x+h)+h\}
$$

is a metric, the Lévy metric, on the set $\Delta$ of all distribution functions $[1,2]$.

We can now state our

Theorem. Let $(S, \mathscr{J}, T)$ be a Menger space under a t-norm $T$ which is such that $\lim T(a, x)=a$ uniformly on the closed interval $[0,1]$; let $S$ be endowed with the $x \rightarrow 1$

$\varepsilon, \lambda$-topology, $S \times S$ with the induced product topology, and $\Delta$ with the topology induced by the Lévy metric. Then the probabilistic distance function $\mathscr{J}$ is a uniformly continuous mapping from $S \times S$ into $\Delta$.

Proof. Let $\varepsilon>0$ be given. Since $T(a, x) \rightarrow a$ uniformly as $x \rightarrow 1$, there exists a $\lambda, 0<\lambda<1$, such that

$$
T(a, 1-\lambda)>a-\varepsilon / 2, \text { for all } a \in[0,1] .
$$

Now let $p, q$ be any pair of points of $S$ and let $p^{\prime} \in N_{p}(\varepsilon / 2, \lambda)$ and $q^{\prime} \in N_{q}(\varepsilon / 2, \lambda)$, so that

$$
F_{p p^{\prime}}(\varepsilon / 2)>1-\lambda \text { and } F_{q q^{\prime}}(\varepsilon / 2)>1-\lambda .
$$

Then for any $x \geqq 0$,

$$
\begin{aligned}
F_{p^{\prime} q^{\prime}}(x+\varepsilon) & \geqq T\left(F_{p^{\prime} q}(x+\varepsilon / 2), F_{q q^{\prime}}(\varepsilon / 2)\right) \\
& \geqq T\left(T\left(F_{p p^{\prime}}(\varepsilon / 2), F_{p q}(x)\right), F_{q q^{\prime}}(\varepsilon / 2)\right) \\
& \geqq T\left(T\left(1-\lambda, F_{p q}(x)\right), 1-\lambda\right) \\
& >T\left(F_{p q}(x), 1-\lambda\right)-\varepsilon / 2 \\
& >F_{p q}(x)-\varepsilon,
\end{aligned}
$$

whence

$$
F_{p q}(x)<F_{p^{\prime} q^{\prime}}(x+\varepsilon)+\varepsilon .
$$

Similarly we have,

$$
F_{p q}(x)>F_{p^{\prime} q^{\prime}}(x-\varepsilon)-\varepsilon .
$$

Combining the two inequalities yields,

$$
L\left(F_{p q}, F_{p^{\prime} q^{\prime}}\right)<\varepsilon
$$

Thus $\mathscr{J}$ is continuous at $(p, q)$; and since $\lambda$ depends only on $\varepsilon$ and not on $p$ and $q$, $\mathscr{J}$ is uniformly continuous and the theorem is proved.

Corollary 1 . If $(S, \mathscr{J}, T)$ is a Menger space under a continuous t-norm $T$, then $\mathscr{J}$ is a uniformly continuous mapping from $S \times S$ into $A$. 
Proof. $T$ is uniformly continuous on the unit square.

Corollary 2. If $(S, \mathscr{J}, T)$ is a Menger space under a t-norm $T$ which is stronger than some continuous t-norm, then $\mathscr{J}$ is a uniformly continuous mapping trom $S \times S$ into $\Delta$.

Corollary $3 . I f(S, \mathscr{J})$ is a Wald space then $\mathscr{J}$ is a uniformly continuous mapping from $S \times S$ into $A$.

Proof. Any Wald space is a Menger space under the continuous $t$-norm Prod given by $\operatorname{Prod}(a, b)=a b[5]$.

The $\varepsilon, \lambda$-topology can also be defined in terms of sequential convergence via: $p_{n} \rightarrow p$ if and only if $F_{p_{n} p} \rightarrow H$ pointwise, where $H$ is the distribution function given by

$$
H(x)= \begin{cases}0, & x \leqq 0 \\ 1, & x>0\end{cases}
$$

or equivalently, if and only if for every $\varepsilon>0$ and every $\lambda>0$, there exists an integer $M(\varepsilon, \lambda)$ such that $p_{n} \in N_{p}(\varepsilon, \lambda)$ whenever $n>M(\varepsilon, \lambda)$. Thus we have the further

Corollary 4. If $p_{n} \rightarrow p$ and $q_{n} \rightarrow q$ then $L\left(F_{p_{n} q_{n}}, F_{p q}\right) \rightarrow 0$.

In addition to the above corollaries, the continuity theorem has further important consequences. One of these, the fact that every PM space has a completion which is unique up to isometry, is the subject of the subsequent paper by H. SHERwooD. Several others follow immediately from Corollary 4 and the well-known fact that for any sequence $\left\{F_{n}\right\}$ of distribution functions the following are equivalent $[1,2]$ :

(1) $L\left(F_{n}, F\right) \rightarrow 0$;

(2) The sequence $\left\{F_{n}\right\}$ converges completely to $F$, i.e., $F_{n}(x) \rightarrow F(x)$ for every $x$ in the continuity set of $F, F_{n}(-\infty) \rightarrow F(-\infty)=0$, and $F_{n}(+\infty) \rightarrow$ $\rightarrow F(+\infty)=1$;

(3) For any function $g$ which is bounded and continuous on $(-\infty, \infty)$,

$$
\int g d F_{n} \rightarrow \int g d F
$$

From Corollary 4 and these equivalences we have at once that, under the hypotheses of the continuity theorem, if $p_{n} \rightarrow p$ and $q_{n} \rightarrow q$ then the sequence of distance distribution functions $\left\{F_{p_{n} q_{n}}\right\}$ converges completely to the distance distribution function $F_{p q}$, and that for any $g$ as in (3),

$$
\int g d F_{p_{n} q_{n}} \rightarrow \int g d F_{p q} .
$$

This last result is not strong enough to yield the convergence of the moments of the $F_{p_{n} q_{n}}$ to the moments of $F_{p q}$ from the convergence of the points $p_{n} \rightarrow p$ and $q_{n} \rightarrow q$. However, as is well-known, under certain further hypotheses (see, e.g. [2]) results of this nature can be obtained.

\section{References}

[1] Gnedenko, B. V., and A. N. Kolmogonov: Limit distributions for sums of independent random variables. Cambridge: Addison-Wesley 1954. 
[2] Lo亡̀ve, M.: Probability theory. New York: D. Van Nostrand 1955.

[3] Menger, K.: Statistical metrics. Proc. Nat. Acad. Sci., U.S.A., 28, 535-537 (1942).

[4] Schweizer, B.: Lectures on probabilistic metric spaces. The University of Arizona 1965.

[5] - and A. Sklar: Statistical metric spaces. Pacific J. Math. 10, 313-334 (1960).

[6] WALD, A.: On a statistical generalization of metric spaces. Proc. Nat. Acad. Sci., U.S.A. 29, 196-197 (1943).

University of Massachusetts

Amherst, Massachusetts 